\title{
Proposta de Nova Curva de Altura Uterina para Gestações entre a 20ํㅡ e a 42ª Semana
}

\author{
Proposal of a New Uterine Height Growth Curve for Pregnancies between 20 and 42 Weeks
}

\author{
Silvio Martinelli, Roberto E. Bittar, Marcelo Zugaib
}

\begin{abstract}
RESUMO
Objetivos: construir uma curva de altura uterina, em função da idade gestacional, em população de gestantes normais. Analisar diferenças com as curvas existentes e avaliar a influência da cor, paridade e peso materno na variação da altura uterina.

Métodos: entre julho de 1997 e julho de 1999, 100 gestantes sem intercorrências clínicas e/ou obstétricas foram submetidas a medidas de altura uterina, da $20^{a}$ à $42^{a}$ semana de gestação. A idade gestacional foi confirmada por ultra-sonografia em todos os casos. O mesmo observador realizou 726 medidas de altura uterina, com fita métrica, da borda superior da sinfise púbica ao fundo uterino.

Resultados: foram obtidas curvas e tabelas de altura uterina em função da idade gestacional. O crescimento da altura uterina foi, em média, $0,7 \mathrm{~cm} / \mathrm{semana}$. Os valores médios de altura uterina deste estudo mostraram-se estatisticamente diferentes, quando comparados a outras curvas de altura uterina. Não foram encontradas diferenças estatisticamente significativas para as variáveis cor, paridade e peso em relação à altura uterina.

Conclusão: a construção de uma curva própria de altura uterina, por meio de metodologia adequada, visa o diagnóstico clínico das alterações do crescimento fetal, fato que deverá ser analisado em estudo posterior.
\end{abstract}

PALAVRAS-CHAVE: Gravidez normal. Crescimento e desenvolvimento fetal. Idade gestacional. Diagnóstico pré-natal.

\section{Introdução}

A gestação pode ser complicada por diversas condições que levam a alterações do crescimento fetal normal. A restrição do crescimento fetal (RCF) é a segunda principal causa de morbidade e mortalidade perinatal, sendo superada apenas pela prematuridade. A mortalidade perinatal é de seis a dez vezes maior para os recém-nascidos com $\mathrm{RCF}$, em relação aos adequados para a idade gestacional. A incidência da RCF é bastante variável, dependendo da população estudada e critérios de definição e diagnóstico empregados. Na Clínica Obstétrica da FMUSP, a incidência média da RCF

Clínica Obstétrica da Faculdade de Medicina da Universidade de São Paulo

Correspondência:

Silvio Martinelli

Rua Peixoto Gomide, 268 apto. 43 - Cerqueira César

01409-000 - São Paulo - SP situa-se em $15 \%{ }^{1}$.

O feto também pode apresentar crescimento exacerbado. A macrossomia é definida quando o peso fetal for maior ou igual a $4.000 \mathrm{~g}^{2}$. A incidência de macrossomia varia entre 0,4 e $10 \%{ }^{3,4}$. Nos fetos macrossômicos, o pólo cefálico fetal, pelo grande tamanho, apresenta menor moldagem, levando a dificuldades de insinuação. Há maior incidência de distocia de ombros, lesão do plexo braquial, fraturas de clavícula e fraturas de úmero. A longo prazo, pode advir incapacidade neurológica grave, resultante do trauma.

A detecção dos desvios de crescimento fetal, por meio de acompanhamento pré-natal adequado, é a melhor forma de prevenir complicações. Com o diagnóstico no período antenatal, medidas poderão ser tomadas com o intuito de reduzir a morbidade e mortalidade perinatais. Apesar de não se dispor ainda de um teste fiel para a identificação de alterações de crescimento fetal, durante a 
evolução da gestação, existem métodos clínicos e ultra-sonográficos que, se utilizados em conjunto, aumentam a possibilidade de se efetuar corretamente esse diagnóstico.

A medida da altura uterina é método clínico utilizado para o acompanhamento do crescimento do feto durante a gestação. Utiliza-se, para este fim, fita métrica, medindo-se a distância, em centímetros, da sínfise púbica ao fundo uterino. Quaranta et al. ${ }^{5}$ e Calvert et al. ${ }^{6}$ demonstraram que, entre 20 e 34 semanas, a altura uterina em centímetros equivale à idade gestacional, em semanas. A bexiga deve estar vazia antes da realização da medida ${ }^{7}$.

Para a avaliação do crescimento fetal, foram construidas curvas relacionando a medida da altura uterina com a idade gestacional. Valores abaixo ou acima de determinado padrão (10 ou 90응 percentil ou um ou dois desvios-padrão) são usados como método de triagem para o diagnóstico de RCF ou macrossomia.

Alguns autores encontraram bons valores preditivos para o diagnóstico da RCF pela medida da altura uterina ${ }^{5,6,8-10}$, ao passo que outros não confirmaram estes achados ${ }^{11,12}$. Para o diagnóstico de macrossomia, a literatura também se mostrou discordante ${ }^{9,12}$.

Alguns autores recomendam que cada população deva ter sua própria curva de altura uterina, dadas as diferenças encontradas entre as curvas já construídas ${ }^{9,13}$. Em nosso meio, são poucos os estudos que relacionam uma curva de crescimento do útero com a idade gestacional ${ }^{14-19}$. Estes estudos não foram uniformes em relação à metodologia empregada, diferindo entre si por vários aspectos: forma de acompanhamento das gestantes, avaliação do peso de nascimento, cálculo da idade gestacional e número de examinadores.

Por estes fatos, este estudo se propõe a construir uma curva de altura uterina, em função da idade gestacional, em gestantes com idade gestacional confiável, sem intercorrências clínicas e/ ou obstétricas, de modo prospectivo e com mensurações efetuadas por um único observador. Com este trabalho também visamos analisar diferenças com as curvas existentes e avaliar a influência da cor, paridade e peso materno na variação da altura uterina.

\section{Pacientes e Métodos}

No período de julho de 1997 a julho de 1999, realizou-se, no Ambulatório da Clínica Obstétrica do Hospital das Clínicas da FMUSP, estudo prospectivo envolvendo 100 gestantes, entre a $20^{\text {a }}$ e $42^{\text {a }}$ semanas de gravidez. Todas as gestantes assinaram termo de consentimento aprovado pelo Conselho de Ética do Hospital das Clínicas da FMUSP.

O critério para o cálculo da idade gestacional foi baseado na data da última menstruação, confirmado por exame ultra-sonográfico, realizado até a $12^{\text {a }}$ semana de gestação ou por, pelo menos, dois exames ultra-sonográficos concordantes, realizados entre a $12^{\text {a }}$ e $20^{a}$ semana de gravidez. Os seguintes critérios de inclusão foram utilizados: ausência de intercorrências clínicas ou obstétricas, parto entre a $37^{\text {a }}$ e $42^{\text {a }}$ semana de gestação e recém-nascidos (RN) com peso compreendido entre os percentis 10 e 90, segundo Ramos ${ }^{20}$. Foram excluídas do estudo as gestantes com gestação gemelar, situação transversa, malformações fetais e polidrâmnio.

O controle pré-natal ocorreu por meio de consultas mensais, até a $32^{\text {a }}$ semana, quinzenais, até a $36^{\mathrm{a}}$ semana, e semanais, até o parto. As gestantes foram orientadas para esvaziamento vesical completo, pelo menos 30 minutos antes do exame. Em seguida foram submetidas a medidas de altura uterina, realizadas por um único profissional, utilizando fita métrica graduada em centímetros.

Colocava-se a gestante em decúbito dorsal horizontal, com os membros inferiores em extensão, posicionando-se a fita métrica sobre o eixo longitudinal do útero. As medidas foram realizadas da borda superior da sinfise púbica ao fundo uterino, utilizando-se a face cubital da mão para fixar a fita, segundo a técnica descrita por Belizán et al. ${ }^{8} \mathrm{~A}$ estimativa dos parâmetros da curva normal foi realizada pelo procedimento mixed do software SAS, versão $6.12^{21}$. Como as medidas de cada gestante eram realizadas em mais de uma oportunidade, a análise estatística considerou esta dependência entre as medidas na mesma gestante (modelos mistos para medidas repetidas). Para a construção da curva foi utilizada uma equação polinomial de segundo grau e o teste dos efeitos realizado pelas somas de quadrados seqüenciais (estatística F tipo I no software SAS) $^{21}$.

Foi também avaliado o efeito da cor, paridade e peso materno na variação da altura uterina, por meio do software $\mathrm{SAS}^{21}$. Para este fim, as gestantes foram subdivididas nos grupos: brancas e não-brancas, nuliparas e multíparas, com peso normal e sobrepeso. Em cada grupo, foi verificado se houve diferença estatisticamente significativa na variação da altura uterina ao longo da gestação.

Para a análise da variação intra-observador (desvio-padrão), foram selecionadas seis gestantes, em diferentes idades gestacionais, que tive- 
ram suas medidas de altura uterina verificadas seis vezes, em esquema de rodizio, pelo mesmo observador.

\section{Resultados}

A idade das 100 gestantes variou de 17 a 39 anos (média de $26 \pm 6$ anos) e em relação à cor, houve predomínio de gestantes de cor branca $(75 \%)$, em relação às cores parda $(11 \%)$ e preta (14\%). Quanto ao estado civil, houve predominio de gestantes com união estável (casadas), em 63 casos $(63 \%)$.

Das gestantes analisadas, $10 \%$ eram tabagistas. O número de cigarros consumidos por dia foi em média de $6 \pm 3$, variando de 2 a 10 . Quanto à paridade, 49 gestantes eram nuliparas (49\%), 29 eram primiparas $(29 \%)$ e 22 tinham dois ou mais partos $(22 \%)$.

Em relação ao tipo de parto, o parto vaginal foi o mais freqüente (69\%), tendo sido o fórcipe indicado em 13 casos (13\%). A cesárea foi realizada em 31 gestantes (31\%), com as seguintes indicações: distocia funcional $(17 \%)$, duas ou mais cesáreas anteriores (5\%), distocia óssea (3\%), mecônio intraparto (3\%), apresentação pélvica $(2 \%)$ e sofrimento fetal agudo (1\%).

Segundo o gráfico de Rosso ${ }^{22}, 47$ gestantes (47\%) foram classificadas como tendo peso normal, 25 gestantes $(25 \%)$ foram classificadas como tendo baixo peso e 28 gestantes (28\%) apresentavam sobrepeso, na primeira consulta pré-natal.

Em relação aos recém-nascidos, a idade gestacional média do parto foi de $39 \pm 1$ semanas e o peso médio de nascimento de $3.221 \pm 319$ gramas. Quanto ao sexo, houve predomínio de RN do sexo feminino (52\%). Em nenhum dos casos analisados, houve diferença maior de 14 dias, entre a idade gestacional calculada e a estimada pelo método de Capurro et al. ${ }^{23}$ Em relação aos índices de Apgar no 1ํ e 5o minuto, não houve valores inferiores a 7 .

Em relação às 100 gestantes estudadas, houve um total de 726 medidas, com uma média de 7,3 medidas por gestante. O início do acompanhamento variou de 20 a 34 semanas, sendo que as gestantes foram acompanhadas até um período máximo que variou de 32 a 42 semanas. O maior número de medidas foi realizado entre 35 e 39 semanas de gestação. O valor da altura uterina variou de um mínimo de $19 \mathrm{~cm}$, no início do estudo, até um máximo de $38 \mathrm{~cm}$, no final, com maior número de medidas correspondendo a $33 \mathrm{~cm}$.

Para a construção da curva, o modelo considerado foi a equação quadrática: $\mathrm{AU}=\beta_{0}+\beta_{1} \mathrm{IG}+$ $\beta_{2} \mathrm{IG}^{2}$, onde AU representa a altura uterina, IG representa a idade gestacional e $\beta_{0}, \beta_{1}$ e $\beta_{2}$ representam os coeficientes de regressão da equação. Após a análise de variância com o teste seqüencial dos efeitos e cálculo dos coeficientes, a equação que representa o crescimento médio da altura uterina $(\mathrm{cm})$ em função da idade gestacional (semanas) pôde ser assim descrita: $\mathrm{AU}=-1,85956+$ 1,32937 IG - 0,01007 IG .

Na Tabela 1, encontram-se os intervalos de confiança em cm com niveis de confiança de 80 , 90, 95 e 99\% da medida da altura uterina para idades gestacionais entre 20 e 42 semanas. Estes intervalos foram representados na Figura 1, por uma curva de altura uterina em relação à idade gestacional, com os percentis 10, 50 e 90 .

$\mathrm{O}$ aumento na medida da altura do útero relacionou-se com a progressão da gestação (Figura 2). O maior aumento ocorreu entre a $20^{\mathrm{a}} \mathrm{e}$ 34a semana (média: 0,7 cm/sem), após o que a taxa de crescimento diminuiu até a $42^{\mathrm{a}}$ semana (média: $0,5 \mathrm{~cm} / \mathrm{sem}$ ). Para cada semana de gravidez, o aumento esperado da altura uterina foi, em média, $0,7 \mathrm{~cm} / \mathrm{sem}$. O valor médio da altura uterina encontrado, após a $37^{\text {a }}$ semana, foi 34,9 $\mathrm{cm}$ (Tabela 2).

Não foram encontradas diferenças estatisticamente significativas para as variáveis cor, paridade e peso em relação à altura uterina. Considerando-se o peso materno, as gestantes com sobrepeso apresentaram altura uterina média 0,4 $\mathrm{cm}$ maior do que as gestantes com peso normal.

O desvio-padrão intra-observador foi estimado como a raiz quadrada da média das variâncias calculadas dentro de cada gestante. O desvio-padrão intra-observador encontrado foi, em média, igual a $1,1 \mathrm{~cm}$.

\section{Discussão}

A medida da altura uterina consiste em método clínico simples e de baixo custo para a avaliação do crescimento fetal. Pode ser utilizada por médicos e paramédicos, com experiência variável, sem que haja perda significativa de sua acurácia $^{6}$. Este método assume ainda maior importância em países em desenvolvimento, onde recursos tecnológicos mais sofisticados para a avaliação do crescimento fetal podem ser escassos, ou mesmo estarem ausentes.

Além do próprio feto, o volume de líquido amniótico, a plenitude vesical, a situação fetal e o excesso ou escassez de tecido celular subcutâneo materno podem interferir na relação entre o peso fetal e a medida da altura uterina. 
Tabela 1 - Valores da altura uterina $(\mathrm{cm})$ nos percentis em relação à idade gestacional (semanas).

\begin{tabular}{|c|c|c|c|c|c|c|c|c|c|}
\hline \multirow{2}{*}{$\begin{array}{l}\text { Idade } \\
\text { gestacional }\end{array}$} & \multicolumn{9}{|c|}{ Percentil } \\
\hline & 0,5 & 2,5 & 5 & 10 & 50 & 90 & 95 & 97,5 & 99,5 \\
\hline 20 & 16,9 & 17,8 & 18,3 & 18,8 & 20,7 & 22,6 & 23,1 & 23,6 & 24,5 \\
\hline 21 & 17,8 & 18,7 & 19,2 & 19,7 & 21,6 & 23,5 & 24,0 & 24,5 & 25,4 \\
\hline 22 & 18,7 & 19,6 & 20,1 & 20,6 & 22,5 & 24,4 & 24,9 & 25,4 & 26,3 \\
\hline 23 & 19,6 & 20,5 & 21,0 & 21,5 & 23,4 & 25,3 & 25,8 & 26,3 & 27,2 \\
\hline 24 & 20,4 & 21,4 & 21,8 & 22,4 & 24,2 & 26,1 & 26,7 & 27,1 & 28,0 \\
\hline 25 & 21,3 & 22,2 & 22,7 & 23,2 & 25,1 & 27,0 & 27,5 & 28,0 & 28,9 \\
\hline 26 & 22,1 & 23,0 & 23,5 & 24,0 & 25,9 & 27,8 & 28,3 & 28,8 & 29,7 \\
\hline 27 & 22,9 & 23,8 & 24,3 & 24,8 & 26,7 & 28,6 & 29,1 & 29,6 & 30,5 \\
\hline 28 & 23,7 & 24,6 & 25,0 & 25,6 & 27,5 & 29,4 & 29,9 & 30,4 & 31,3 \\
\hline 29 & 24,4 & 25,3 & 25,8 & 26,3 & 28,2 & 30,1 & 30,6 & 31,1 & 32,0 \\
\hline 30 & 25,2 & 26,1 & 26,5 & 27,1 & 29,0 & 30,8 & 31,4 & 31,8 & 32,8 \\
\hline 31 & 25,9 & 26,8 & 27,3 & 27,8 & 29,7 & 31,6 & 32,1 & 32,6 & 33,5 \\
\hline 32 & 26,6 & 27,5 & 27,9 & 28,5 & 30,4 & 32,2 & 32,8 & 33,2 & 34,2 \\
\hline 33 & 27,2 & 28,2 & 28,6 & 29,2 & 31,0 & 32,9 & 33,5 & 33,9 & 34,8 \\
\hline 34 & 27,9 & 28,8 & 29,3 & 29,8 & 31,7 & 33,6 & 34,1 & 34,6 & 35,5 \\
\hline 35 & 28,5 & 29,4 & 29,9 & 30,4 & 32,3 & 34,2 & 34,7 & 35,2 & 36,1 \\
\hline 36 & 29,2 & 30,1 & 30,5 & 31,1 & 32,9 & 34,8 & 35,4 & 35,8 & 36,7 \\
\hline 37 & 29,7 & 30,7 & 31,1 & 31,7 & 33,5 & 35,4 & 36,0 & 36,4 & 37,3 \\
\hline 38 & 30,3 & 31,2 & 31,7 & 32,2 & 34,1 & 36,0 & 36,5 & 37,0 & 37,9 \\
\hline 39 & 30,9 & 31,8 & 32,2 & 32,8 & 34,7 & 36,6 & 37,1 & 37,6 & 38,5 \\
\hline 40 & 31,4 & 32,3 & 32,8 & 33,3 & 35,2 & 37,1 & 37,6 & 38,1 & 39,0 \\
\hline 41 & 31,9 & 32,8 & 33,3 & 33,8 & 35,7 & 37,6 & 38,1 & 38,6 & 39,5 \\
\hline 42 & 32,4 & 33,3 & 33,8 & 34,3 & 36,2 & 38,1 & 38,6 & 39,1 & 40,0 \\
\hline
\end{tabular}

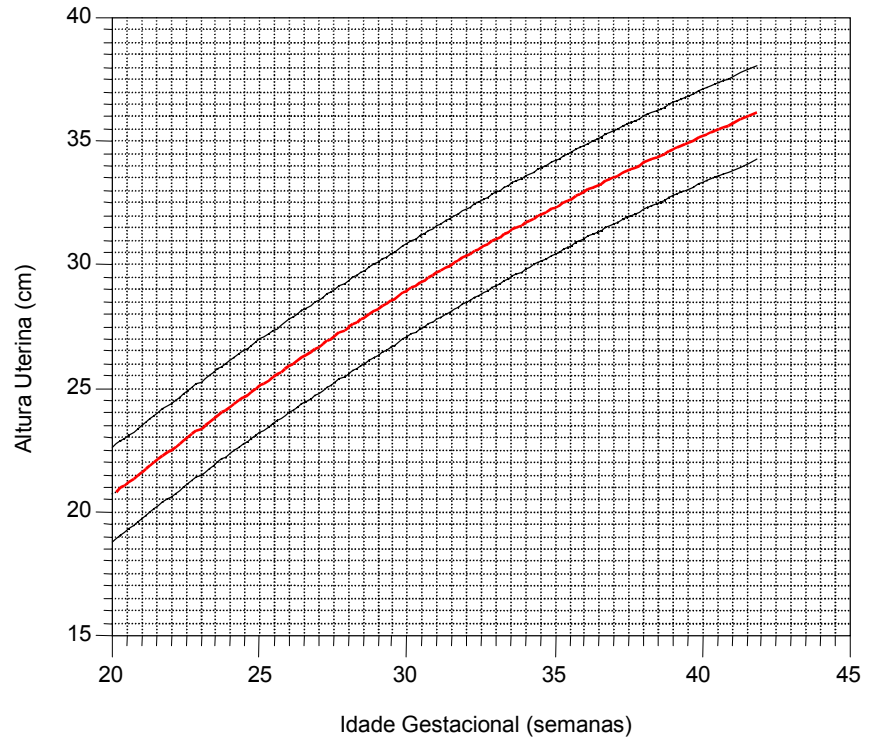

Figura 1 - Curva de valores da altura uterina: percentis 10, 50 e 90 (nível de confiança de $80 \%)$.

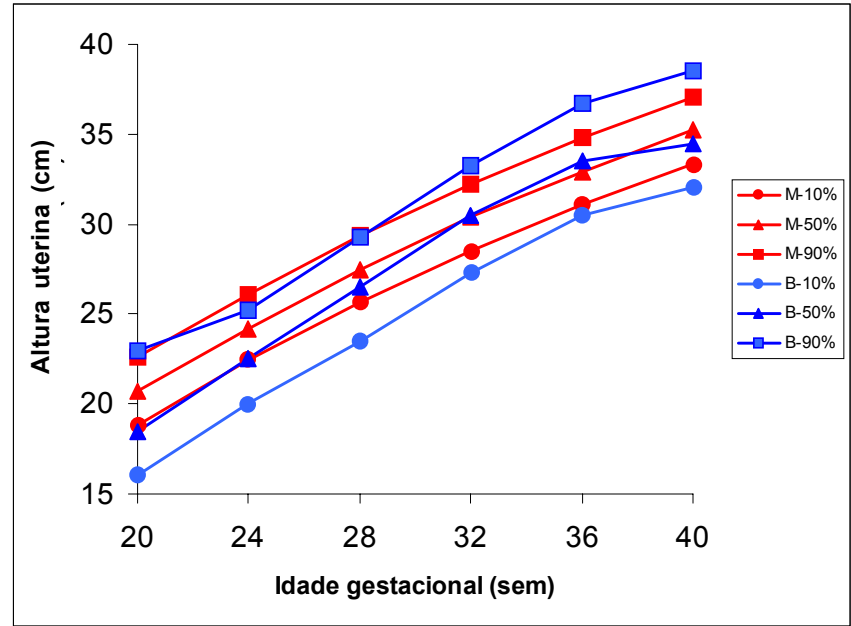

Figura 2 - Comparação entre curvas de altura uterina: presente estudo (M) e Belizán et al. ${ }^{8}$ (B) - percentis 10,50 e 90. 
Tabela 2 - Comparação entre valores de medidas de altura uterina em vários estudos (percentil 50), segundo a técnica de Belizán et al. ${ }^{8}$

\begin{tabular}{|c|c|c|c|c|c|c|}
\hline \multicolumn{7}{|c|}{ Medidas de altura uterina $(\mathrm{cm})$} \\
\hline \multirow[t]{2}{*}{ Estudos } & \multicolumn{6}{|c|}{ Idade gestacional (semanas) } \\
\hline & 20 & 24 & 28 & 32 & 36 & 40 \\
\hline Belizán et al., 1978 (Argentina) ${ }^{8}$ & 18,5 & 22,5 & 26,5 & 30,5 & 33,5 & 34,5 \\
\hline Quaranta et al., 1981 (Inglaterra) ${ }^{5}$ & 20,2 & 24,1 & 28,1 & 31,8 & 34,7 & 36,3 \\
\hline Calvert et al., 1982 (Inglaterra) ${ }^{6}$ & 18,8 & 22,9 & 26,8 & 30,2 & 33,7 & 36,2 \\
\hline Taylor et al., 1984 (Australia)29 & 18,0 & 23,0 & 28,0 & 31,0 & 34,0 & 36,5 \\
\hline Mathai et al., 1987 (Índia)24 & & 21,5 & 24,5 & 27,5 & 31,5 & 33,5 \\
\hline Azziz et al., 1988 (EUA) $)^{25}$ & 20,5 & 24,6 & 28,3 & 32,8 & 36,5 & 39,8 \\
\hline Steingrímsdóttir et al., 1995 (Suécia) $^{28}$ & 19,0 & 23,1 & 27,1 & 30,1 & 33,6 & 35,8 \\
\hline Silva et al., 1986 (Brasil) ${ }^{15}$ & 19,0 & 23,0 & 26,0 & 30,0 & 32,5 & 34,0 \\
\hline Barini, 1989 (Brasil) $)^{17}$ & 18,5 & 22,2 & 25,9 & 29,3 & 32,2 & 34,2 \\
\hline Presente estudo, 2000 (Brasil) & 20,7 & 24,2 & 27,5 & 30,4 & 32,9 & 35,2 \\
\hline
\end{tabular}

A maioria dos trabalhos que utilizam esta metodologia para construir uma curva-padrão de altura uterina estão restritos a gestantes sem intercorrências clínicas e de peso e estatura adequados. Alguns autores não fizeram correções quanto à obesidade, situação fetal e volume de líquido amniótico $0^{5,24,25}$. Excluímos de nossa casuística as gestantes com intercorrências clínicas, com gemelaridade, situação transversa, malformações fetais e polidrâmnio, para evitar que estes fatores interferissem na avaliação do crescimento fetal.

As gestantes com sobrepeso, classificadas segundo o critério de Rosso ${ }^{22}$, não foram excluídas do nosso estudo. Assim como descrito por Indira et al. ${ }^{26}$ e Euans et al. ${ }^{27}$, não encontramos diferença estatisticamente significativa entre a variação da altura uterina e o sobrepeso materno. A diferença encontrada em nosso estudo, entre a medida da altura uterina do grupo com sobrepeso e do grupo com peso normal, foi de apenas $0,4 \mathrm{~cm}$, em média. Steingrimsdóttir et al. ${ }^{28}$ encontraram diferença média de $2,0 \mathrm{~cm}$, ao longo da gestação, entre o grupo de gestantes mais magras (peso inferior a $53 \mathrm{~kg}$ ) e mais obesas (peso superior a 79 $\mathrm{kg})$.

A exemplo dos relatos de Belizán et al. ${ }^{8} \mathrm{e}$ Taylor et al. ${ }^{29}$, não encontramos diferença significativa da altura uterina entre os grupos de gestantes nuliparas e multíparas. A cor também não foi fator determinante na variação de nossas medidas, assim como descrito no estudo de Euans et al. ${ }^{27}$ Não comparamos as gestantes quanto ao hábito de fumar, pois nosso grupo de tabagistas correspondeu a apenas $10 \%$ dos casos, e o número de cigarros consumido foi, em média, de 6.

Para a construção de curvas-padrão de altura uterina, observam-se metodologias diferentes adotadas pelos diversos autores em relação ao número de gestantes estudadas, tipo de estudo (prospectivo ou retrospectivo), cálculo da idade gestacional e número de examinadores.

Nossa casuística consistiu de 100 gestantes avaliadas por meio de 726 medidas de altura uterina. Esses dados são comparáveis a vários outros relatos, que mostraram número semelhante ou menor de gestantes estudadas ${ }^{5,11,13,26}$. Neste estudo, optamos pelo acompanhamento prospectivo, concordando com a maior parte dos trabalhos internacionais que construíram uma curva de normalidade para a altura uterina. Dentre os estudos nacionais, apenas dois foram realizados prospectivamente ${ }^{16,18}$. O critério utilizado para o cálculo da idade gestacional foi a data da última menstruação, confirmada por ultrasonografia até a $12^{\mathrm{a}}$ semana de gestação, ou por, pelo menos, dois exames ultra-sonográficos concordantes, realizados entre a $12^{\mathrm{a}}$ e $20^{\mathrm{a}}$ semana de gravidez. Poucos ensaios utilizaram a ultrasonografia para a confirmação da idade gestacio$\mathrm{nal}^{6,25,28}$. Apenas um estudo nacional, na literatura pesquisada, utilizou a ultra-sonografia para o cálculo da idade gestacional em todos os $\operatorname{casos}^{16}$.

Todas as nossas medidas foram realizadas por um único observador, fato que determinou uma menor variabilidade. Foram publicados poucos estudos de curvas-padrão de altura uterina com as medidas realizadas pelo mesmo examinador ${ }^{16,18,29}$.

A variação intra-observador (desvio-padrão) por nós encontrada foi em média $1,1 \mathrm{~cm}$. Calvert et al. ${ }^{6}$ investigaram a reprodutibilidade da medida da altura uterina em 12 gestantes, com seis medidas realizadas por seis diferentes examinadores e a variação intra-observador foi de $1,24 \mathrm{~cm}$. Bagger et $a .^{30}$ analisaram o desempenho de cinco examinadores, em que cada um fazia três medi- 
das de altura uterina em 33 gestantes, no terceiro trimestre, e o coeficiente intra-observador encontrado foi, em média, de 1,5 a 2,0 cm.

Deve-se considerar, ao interpretar os valores de altura uterina, que além dos fatores até então relatados, estes dependem também da técnica utilizada para a realização da medida. As medidas são realizadas da borda superior da sinfise púbica ao fundo uterino, fixando-se a fita métrica de diferentes formas. Fescina et al. ${ }^{11}$ indicam que a leitura deve ser realizada com a fita entre os dedos indicador e médio. Segundo Belizán et al. ${ }^{8}$, utiliza-se a face cubital da mão para fixar a fita, e por isso, ao descrever uma curva maior, o valor que se obtém é 1,5 $\pm 0,6 \mathrm{~cm}$ maior em relação ao obtido com a técnica anterior, no terceiro trimestre da gestação ${ }^{31}$. Ainda, a fita pode ser fixada entre os dedos polegar e indicador ${ }^{18}$.

A técnica da medida da altura uterina realizada em nosso estudo foi a descrita por Belizán et al. ${ }^{8}$, utilizada pela maioria dos trabalhos descritos na literatura que se propuseram a construir uma curva de normalidade.

Na Tabela 2, apresentamos uma comparação entre os valores médios de altura uterina (percentil 50) entre vários estudos. A curva construída na Índia ${ }^{24}$ foi a que apresentou os menores valores, enquanto que a curva proveniente dos Estados Unidos da América ${ }^{25}$, os maiores valores. A diferença entre estas duas curvas, na $40^{a}$ semana de gestação, atinge o valor de $6,3 \mathrm{~cm}$. Comparando nossas medidas com as de estudos nacionais ${ }^{15,17}$, observamos diferenças superiores a $1,0 \mathrm{~cm}$, em várias idades gestacionais. Não foi possivel a comparação com os outros trabalhos nacionais, pois foram utilizadas outras técnicas para a medida da altura uterina.

A análise comparativa da curva descrita por Belizán et $a .^{8}$ com a nossa mostrou que estas são diferentes. Considerando-se o percentil 50, na $20^{\mathrm{a}}$ semana, nossa curva apresenta valor de altura uterina superior, em média, de $2,2 \mathrm{~cm}$. Com o progredir da gestação, esta diferença diminui até estar ausente na $32^{\mathrm{a}}$ semana. Após este período, até a 36 semana de gestação, a curva de Belizán et al. ${ }^{8}$ apresenta valores maiores em relação à nossa curva, para posteriormente sofrer uma horizontalização até o final da gestação, mostrando novamente valores inferiores, na $40^{\text {a }}$ semana de gravidez (diferença média: 0,7 cm). Por meio de análise estatística, utilizando um intervalo de confiança de 99\%, ao compararmos as duas curvas médias, estas diferem significativamente entre a $20^{\underline{a}}$ e $29^{a}$ semana e após a 40a semana (Figura 2).

Diante desses resultados, observamos que há grandes diferenças entre os valores de altura uterina utilizados em diferentes países, e até mesmo dentro de um mesmo país. Isto pode ser explicado, em parte, pelas diferentes metodologias utilizadas para a construção das curvas. Também é inegável que grande parte dessas diferenças sejam devidas à diversidade étnica entre as várias populações, além de condições socioeconômicas e nutricionais próprias.

Com o presente estudo, por meio de metodologia adequada, construímos uma curva de altura uterina, em função da idade gestacional, com percentis 10,50 e 90 , em gestantes sem intercorrências clínicas e/ou obstétricas. A sua acurácia quanto ao diagnóstico clínico das alterações do crescimento fetal deverá ser avaliada em estudo posterior.

\section{SUMMARY}

Purpose: to create a uterine height growth curve, according to gestational age, to verify differences among the existing curves and to evaluate the influence of color, parity and maternal weight on the variation of uterine height.

Methods: during the period from July 1997 to July 1999, 100 normal pregnant women were submitted to uterine height measurements between the 20th and 42nd week of gestation. All the pregnant women had ultrasonically confirmed gestational age. A total of 726 measurements of uterine height were carried out by the same examiner, using a metric tape from the upper border of the symphysis pubis to the fundus uteri.

Results: curves and tables of uterine height according to gestational age were obtained. The average uterine height growth was $0.7 \mathrm{~cm} /$ week. The study revealed different average uterine height values in relation to other uterine height growth curves. No statistically significant variations were found between the distributions of uterine heights according to color, parity and weight.

Conclusion: the construction of a methodologically accepted uterine height growth curve aimed to detect, as a clinical method, the fetal growth disturbances. This should be analyzed in a posterior study.

KEY WORDS: Normal pregnancy. Fetal development. Gestational age. Prenatal diagnosis.

\section{Referências}

1. Bittar RE. Crescimento intra-uterino retardado. In: Zugaib M, editor. Medicina Fetal. São Paulo: Atheneu; 1998. p.209-13.

2. Reece LA, Friedman AM, Copel J, Kleinman CS. Prenatal diagnosis and management of deviant 
fetal growth and congenital malformations. In: Reece EA, Constan DR, editors. Diabetes Mellitus in Pregnancy. 2a ed. New York: Churchill Livingstone Inc; 1995. p.219-49.

3. Mathias L,Martins JA, Faccioli R, Naomi E, Carrara W, Neme B. Macrossomia fetal: estudo de 114 casos. Ginecol Obstet Bras 1983; 6:51-60.

4. Spellacy WN, Miller S, Winegar A, Peterson PQ. Macrosomia: maternal characteristics and infant complications. Obstet Gynecol 1985; 66:158-61.

5. Quaranta P, Currell R, Redman CW, Robinson JS. Prediction of small-for-dates infants by measurement of symphysial-fundal-height. $\mathrm{Br} \mathrm{J}$ Obstet Gynaecol 1981; 88:115-9.

6. Calvert JP, Crean EE, Newcomb RG, Pearson JF. Antenatal screening by measurement of symphysis-fundus height. Br Med J (Clin Res Ed) $1982 ; 285: 846-9$.

7. Engstrom JL, Ostrenga KG, Plass RV, Work BA. The effect of maternal bladder volume on fundal height measurements. Br J Obstet Gynaecol 1989; 96:98791.

8. Belizán J, Villar J, Nardin JC, Malamud J, De Vicurna LS. Diagnosis of intrauterine growth retardation by a simple clinical method: measurement of uterine height. Am J Obstet Gynecol 1978; 131:643-6.

9. Grover V, Usha R, Kalra S, Sachdeva S. Altered fetal growth: antenatal diagnosis by symphysis fundal height in India and comparison with western charts. Int J Gynaecol Obstet 1991; 35:231-4.

10.Mohanty C, Das BK, Mishra OP. Parturient fundal height as a predictor of low birth weight. J Trop Pediatr 1998; 44:222-4.

11.Fescina RH, Quevedo C, Martell M, Nieto F, Schwarcz R. La medida de la altura uterina como método sencillo para predecir el crecimiento fetal. Bol Oficina Sanit Panam 1984; 96:377-86.

12. Stuart JM, Healy TJ, Sutton M, Swingler GR. Symphysis-fundus measurements in screening for small-for-dates infants: a community based study in Gloucestershire. J R Coll Gen Pract 1989; 39:458.

13.Pommier M, Escobedo F, Lowenberg E. Estudio de la altura del fondo uterino para la detección del crecimiento intrauterino retardado. Ginecol Obstet Mex 1979; 46:253-9.

14. Cunha SP, Ribeiro JU, Berezowski AT, Duarte G. Evolução da altura uterina e circunferência abdominal em gestantes normais. Rev Paul Med 1985; 103:231-4.

15. Silva JLP, Pereira B, Barini R, Reis C, Faúndes A. Avaliação da curva de crescimento uterino na detecção de recém-nascidos pequenos para a idade gestacional. Ginecol Obstet Bras 1986; 9:323-6.

16.Pedrosa de Freitas CB. Evaluación de la altura uterina durante la gravidez. Rev Latinoam Perinatol 1986; 6:128-32.

17.Barini R. Avaliação da curva de crescimento da altura uterina como método para estimar o peso fetal [tese]. Campinas: Universidade Estadual de Campinas; 1989.

18. Gouveia VL, Reis AFF, Amim Júnior J, Silva VL. Valores normais da medida da altura do fundo de útero na gestação de 20 a 39 semanas. J Bras Ginecol 1993; 103:11-8.

19.Pereira A, Gropen Júnior C, Lage EM, Cabral ACV. Curva de crescimento da medida do útero-fita em gestações de risco habitual acompanhadas no Hospital das Clinicas - UFMG. J Bras Ginecol 1997; 107:297-9.

20.Ramos JLA. Avaliação do CIUR por medidas antropométricas do recém-nascido [tese]. São Paulo: Universidade de São Paulo; 1983.

21.Littell RC, Milliken GA, Stroup WW, Wolfinger R. SAS system for mixed models [software]. Cary: SAS Institute; 1996.

22.Rosso P. A new chart to monitor weight gain during pregnancy. Am J Clin Nutr 1985; 41:644-52.

23. Capurro H, Konichezky S, Fonseca D, CaldeyroBarcia R. A simplified method for diagnosis of gestacional age in the newborn infant. J Pediatr 1978; 93:120-2.

24. Mathai M, Jairaj P, Muthurathnam S. Screening for light-for-gestacional age infants: a comparison of three simple measurements. Br J Obstet Gynaecol 1987; 94:217-21.

25.Azziz R, Smith S, Fabro S. The development and use of a standard symphysial-fundal height growth curve in the prediction of small for gestational age neonates. Int $\mathrm{J}$ Gynaecol Obstet 1988; 26:81-7.

26.Indira R, Oumachigui A, Narayan KA, Rajaram P, Ramalingam G. Symphysis-fundal height measurement: a reliable parameter for assessment of fetal growth. Int J Gynaecol Obstet 1990; 33:1-5.

27.Euans DW, Connor PD, Hahn RG, Rodney WM, Arheart KL. A comparison of manual and ultrasound measurements of fundal height. J Fam Pract 1995; 40:233-6.

28. Steingrimsdóttir T, Cnattingius S, Lindmark G. Symphysis-fundus height: construction of a new Swedish reference curve, based on ultrasonically dated pregnancies. Acta Obstet Gynecol Scand 1995; 74:346-51.

29.Taylor P, Coulthard AC, Robinson JS. Symphysialfundal height from 12 weeks' gestation. Aust N Z J Obstet Gynaecol 1984; 24:189-91.

30.Bagger PV, Sindberg Eriksen P, Secher NJ, Thisted J. Westergaard L. The precision and accuracy of symphysis-fundus distance measurements during pregnancy. Acta Obstet Gynecol Scand 1985; 64:371-4.

31.Schirmer J, Parras AP, Leocádio E, Formiga JFN, Fajardo ML, Costa MM. Medida da altura uterina/ acompanhamento do crescimento fetal. In: Schirmer J, editor. Assistência Pré-Natal: manual técnico. Brasília: Ministério da Saúde; 2000. p.3942 . 